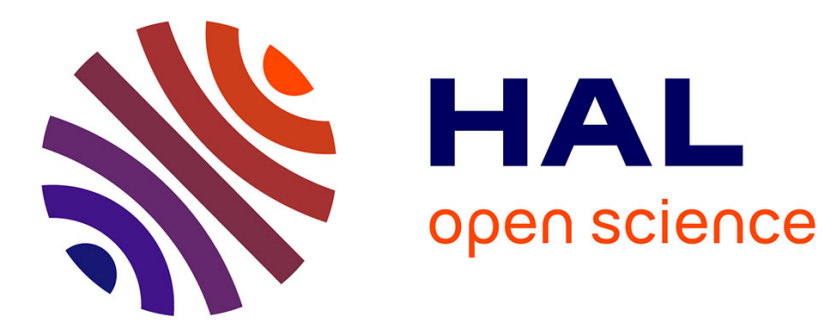

\title{
Pour et la construction du domaine de validation
}

Lise Hamelin, Yukiyo Homma

\section{To cite this version:}

Lise Hamelin, Yukiyo Homma. Pour et la construction du domaine de validation. Faits de langues, 2015. hal-03391770

\author{
HAL Id: hal-03391770 \\ https://hal.science/hal-03391770
}

Submitted on 21 Oct 2021

HAL is a multi-disciplinary open access archive for the deposit and dissemination of scientific research documents, whether they are published or not. The documents may come from teaching and research institutions in France or abroad, or from public or private research centers.
L'archive ouverte pluridisciplinaire HAL, est destinée au dépôt et à la diffusion de documents scientifiques de niveau recherche, publiés ou non, émanant des établissements d'enseignement et de recherche français ou étrangers, des laboratoires publics ou privés. 


\title{
Pour et la construction du domaine de validation
}

\author{
Lise Hamelin* et Yukiyo Homma**
}

Le présent article s'inscrit dans le cadre d'une proposition de représentation des prépositions du français contemporain utilisant les outils de la Théorie des Opérations Prédicatives et Énonciatives (cf. Culioli, 1990, 1999a et 1999b). Dans ce cadre théorique, on considère que l'énoncé construit le sens par une suite d'opérations de repérage des termes qu'il met en jeu les uns par rapport aux autres et par rapport à une situation repère instanciée par l'énonciateur (la personne qui prend en charge l'énoncé) et le moment d'énonciation. Notre analyse fait suite à d'autres travaux s'inscrivant dans ce cadre, notamment la thèse de Homma (2009), consacrée à l'étude des marqueurs en, dans, par et pour en français contemporain, mais également à une série d'articles sur la préposition en anglais (parmi lesquels Gilbert 2000 sur for et Hamelin sur to, for et according to, 2010a), qui ont montré que la position du syntagme prépositionnel pouvait s'avérer pertinente pour l'analyse du fonctionnement du marqueur, en particulier lorsque le SP s’insère dans l'énoncé en position détachée.

Neveu propose les remarques suivantes à propos du détachement :

[le détachement] sert à la fois à décrire tout à la fois : (i) un phénomène très général de discontinuité syntaxique, qui, selon les approches et les types de segments visés, présuppose ou non une conception dérivationnelle de la forme des constructions, autrement dit la dislocation d'un segment linéaire ; (ii) certains faits relatifs aux positions syntaxiques (périphérie propositionnelle, extraposition, incidence, etc.) ;

(iii) les opérations linguistiques dont ces faits sont censés résulter (prédication seconde, topicalisation, thématisation, référenciation, etc.) (Neveu, 2004:100)

L'étude proposée ici consiste en l'analyse de quelques emplois de pour, dans lesquels ce marqueur entretient des rapports privilégiés avec les phénomènes de détachement. Les quatre énoncés ${ }^{1}$ suivant sont représentatifs de ces emplois :

(1) Pour dire les choses clairement ${ }^{2}$, le sentiment nationaliste grandit.

(2) Pour lui, Madame Caillaux n’avait pas eu tort de tirer un coup de révolver sur le Directeur du Figaro.

\footnotetext{
*Université de Caen Basse-Normandie, CRISCO (EA 4255). Courriel : Lise.hamelin@ucergy.fr

** Université Paris Ouest Nanterre la Défense, MoDyCo (UMR 7114). Courriel : yukiyof@hotmail.com

${ }^{1}$ La plupart des exemples sont tirés de la base de données FRANTEXT. Quelques énoncés proviennent de la Toile.

${ }^{2}$ Cet emploi se distingue des autres dans la mesure où le marqueur pour n'introduit par un $\mathrm{SN}$ mais un groupe infinitif.
} 
(3) Pour le tourisme, ce pays est en retard.

(4) Pour l'instant, il attend.

L'emploi illustré en (1) est un commentaire métalinguistique de l'énonciateur sur son propre énoncé, la relation prédicative qui suit, et se satisfait de la glose (1') :

(1') Dire que le sentiment nationaliste grandit, c'est dire les choses clairement / c'est avoir un discours clair.

En (2), le syntagme prépositionnel introduit la source de point de vue qui domine la relation prédicative, ce qui est souligné dans la glose (2') :

(2') Il pensait que Madame Caillaux n'avait pas eu tort de tirer un coup de révolver sur le Directeur du Figaro.

En (3), pour introduit le thème du discours, ce dont il est question dans la relation prédicative qui suit :

(3’) En / Pour ce qui concerne le tourisme, ce pays est en retard.

En (4), le syntagme prépositionnel localise l'événement dénoté par la relation prédicative dans le temps :

(4') Actuellement / En ce moment, il attend.

Les hypothèses défendues dans l'analyse de ces quatre types d'emplois seront que :

- le syntagme en pour construit le domaine (temporel, subjectif, etc.) dans lequel la relation prédicative est validée ;

- ce domaine est systématiquement construit selon une relation d'altérité à un autre domaine (ou à une classe de domaines).

L'analyse proposée mettra en œuvre les concepts de la TOPE. Ce cadre théorique s'est récemment tourné vers le domaine des marqueurs prépositionnels et leur a consacré une grande variété d'analyses. Nous pensons notamment aux travaux de Franckel et Paillard pour le français, et à ceux de Dufaye et de Gilbert $^{3}$ pour l'anglais. Ces études utilisent de façons différentes l'outillage mis à disposition par la théorie. Toutefois, elles ont en commun de considérer les prépositions comme des marqueurs établissant une relation entre un terme repère et un terme repéré. On considérera que le terme repère est le terme introduit par pour et le terme repéré, la relation prédicative qui suit.

Hamelin (2010a) a proposé une analyse en termes de hiatus entre les termes mis en relation par pour, $\mathrm{X}$ (le terme du co-texte gauche) et Y (le terme du cotexte droit), pour les valeurs causale et finale du marqueur. Nous maintiendrons ici cette analyse, mais en faisant l'hypothèse, comme nous venons de l'indiquer, que la position détachée permet, dans les cas observés dans ces pages, la

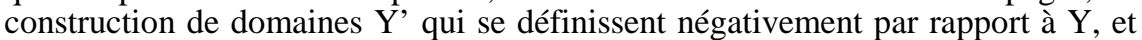
que c’est entre Y et Y' qu'intervient l'altérité.

${ }^{3}$ Cf. bibliographie. 


\section{LES ÉnONCÉS DU TYPe : Pour diRE Les CHOSES ClAIREMENT, LE SENTIMENT} NATIONALISTE GRANDIT.

Pour le démontrer, revenons à l'énoncé (1), dont nous avons dit qu'il se satisfaisait de la glose (1'). On pourra également analyser l'énoncé (5) et sa glose (5’) :

(5) Le salariat, c'est bien sûr l'ensemble des salariés, c'est-à-dire tout ceux qui pour parler comme Marx - vendent leur force de travail ou - pour parler comme le droit civil - louent leurs services moyennant salaire.

(5') Marx dirait que le salariat, c'est tout ceux qui vendent leur force de travail ; là où le droit civil dirait qu'il s'agit de ceux qui louent leurs services moyennant salaire.

Dans les énoncés du type de (1) et (5), pour introduit un groupe infinitif Y caractérisant le type de discours apparaissant dans la proposition X. Y comporte toujours un procès renvoyant à un acte de parole ou de communication, le plus souvent associé à un adverbe de manière ou à l'expression d'une comparaison au moyen de comme. Dans les contextes de cet ordre, Y constitue un commentaire sur $\mathrm{X}$, la relation prédicative, à laquelle il attribue certaines propriétés. Ce sont ces propriétés qui apparaissent sous la forme de l'adverbe clairement en (1) ou des syntagmes comme Marx et comme le droit civil en (5), comme on peut l'observer dans les gloses (1') et (5').

Dans le même temps, on s'aperçoit que ce type d'incise introduit une rupture avec un élément qui peut correspondre au contexte avant ou à un autre énoncé. Pour dire les choses clairement implique, en effet, que, jusque là, les choses n'étaient pas claires, ou auraient pu ou dû l'être davantage. X se démarque de ce qui a été dit précédemment en ce que ce terme se voit attribuer par l'énonciateur la propriété <être clair>. De même, lorsque l'on a pour parler comme quelqu'un, l'énonciateur indique que les propriétés de $X$ en font un énoncé qui aurait pu être pris en charge par la personne en question, ou qui est typique de cette personne. Cela rend $\mathrm{X}$ distinguable des énoncés potentiellement, habituellement, ou précédemment pris en charge par l'énonciateur ${ }^{4}$.

Par ailleurs, on rencontre régulièrement des cas dans lesquels le terme Y met en jeu une comparaison de supériorité (comme en (6) et (7) ci-dessous), qui implique aussi une forme de rupture avec le discours qui précède :

(6) Pour dire les choses plus clairement, j'étudie le comportement de particules qui sont dans la magnétosphère interne [...]

(7) En 1841, Edward Wakefield négocia l'achat de 16000 hectares ou, pour dire les choses plus crûment, arnaqua les Maoris qui durent se contenter de quelques «cadeaux».

\footnotetext{
${ }^{4}$ Lors de la présentation de ce travail au colloque Prépositions et Aspectualité, Agnès Steuckardt souligne, à raison, que cet emploi n'est pas exempt d'une forme de visée. Celle-ci est liée au fait que pour introduit un groupe infinitif mettant en jeu un procès agentif dont le sujet est identifiable à l'énonciateur. Dans ce cas, il s'agit pour l'énonciateur de prendre en charge un énoncé imitant un modèle ou satisfaisant à certains critères, définis par le terme Y.
} 
Pour dire les choses plus clairement / crûment implique que l'énoncé précédent possédait les propriétés <être clair / cru> à un degré inférieur à celui auquel $\mathrm{X}$ les possède. On observe donc la mise en place d'une échelle de degrés qui va de pair avec la dissociation de $X$ et d'un autre énoncé n'ayant pas les propriétés définies en $\mathrm{Y}$, ou les possédant à un degré moindre.

On mettra dans la même catégorie les énoncés dans lesquels apparaît pour dire les choses autrement, qui mettent explicitement en place une forme d'altérité, portant ici sur la formulation de X :

(8) Pour dire les choses autrement, la richesse ne répond pas à une définition conceptuelle objective, elle est d'abord une question de regard.

On peut donc considérer que dans ce type de contextes, pour introduit une forme d'altérité sur l'énonciation, altérité qui se présente sous la forme d'un commentaire métalinguistique (Y) impliquant un phénomène de dissociation par rapport à une prise en charge antérieure ou habituelle, caractéristique de l'énonciateur.

De fait, on ne trouve pas en $\mathrm{Y}$ de caractérisation du discours qui constituerait une forme de qualification de $\mathrm{X}$ n'impliquant pas, au moins implicitement, la prise en compte d'une altérité. Ainsi, sauf détour contextuel particulier, on n’aura pas ( $\left.9^{\prime}\right)$ :

(9) Je ne puis résumer ici sa plaidoirie ; pour parler franchement, mes souvenirs sur cette fin d'audience sont un peu brouillés.

(9’) ?Je ne puis résumer ici sa plaidoirie ; pour parler français, mes souvenirs sur cette fin d'audience sont un peu brouillés.

Par contre, (10) ne fait pas problème, dans la mesure où l'énonciateur annonce très clairement un changement de registre, de type de discours, entre ce qui a été dit précédemment et $\mathrm{X}$, ce qui, dans le contexte, permet l'ironie :

(10) J'écris ceci à la frontière de l'injustice où me pousse la fureur d'une espérance déçue. Hier, j'avais affaire à l'oto-rhino-laryngologiste. Pour parler le jargon, mon exéat était suspendu au nihil obstat de son satisfecit. Un rien. Mais il suffisait que ce spécialiste m’eût précisément dans le nez.

Il apparaît donc que ce qui permet l'emploi de pour que nous envisageons ici est sa capacité à contraster, capacité que l'on retrouve dans d'autres emplois entretenant des affinités particulières avec les phénomènes de détachement.

\section{LES ÉNONCÉS DU TYPE : POUR LUI, MADAME CAILlAUX N'AVAIT PAS EU TORT DE TIRER UN COUP DE RÉVOLVER SUR LE DIRECTEUR DU FIGARO.}

Cette capacité à contraster, ce hiatus entre $\mathrm{Y}$ et $\mathrm{Y}^{\prime}$, se retrouve, en effet, dans les énoncés dans lesquels pour introduit un ou plusieurs animés humains conçu(s) comme la source du point de vue portant sur la relation prédicative $\mathrm{X}$, comme en (2), ou en (11) : 
(11) Grand-mère jugeait ces pluies ineptes. Pour elle, il devait pleuvoir une fois pour toutes et qu'on n'en parle plus.

(11') Grand-mère jugeait ces pluies ineptes. Elle estimait qu'il devait pleuvoir une fois pour toutes et qu'on n'en parle plus.

(11') et (2') font apparaître que $\mathrm{X}$ est validé dans le domaine des représentations qui sont associées aux référents de Madame Caillaux (2) ou de Grand-mère (11).

On peut noter que le statut de sujet-point de vue du référent de $\mathrm{Y}$ est étroitement lié à la nature des relations prédicatives que l'on rencontre dans ce type de contexte. Il s'agit de relations dans lesquelles la délimitation qualitative de l'un des termes est particulièrement saillante. En effet, dans ces énoncés, on a massivement des prédicats statifs avec des procès à fonctionnement compact. D'ailleurs, lorsque la relation prédicative renvoie à un processus dynamique, le SP s'interprète comme délimitant le thème, le topic de l'énoncé, et non le point de vue de référence portant sur cet énoncé. C'est le cas en (12), qu'on peut comparer à (12'), dans lequel on a substitué quant à à pour :

(12) L'armée en prit quelques-uns et la police quelques autres. Pour moi, je restai seul, à côté de Tess et de mes pots de couleurs desséchées.

(12') L'armée en prit quelques-uns et la police quelques autres. Quant à moi, je restai seul, à côté de Tess et de mes pots de couleurs desséchées.

Dans cet énoncé, on indique d'abord ce qui arrive aux autres (quelques uns, quelques autres) avant de se tourner vers l'énonciateur (moi). Dans ce cas, on observe simplement le passage d'un thème à un autre, et le référent de moi n'acquiert pas le statut de sujet-point de vue. Il constitue simplement ce dont on parle, ce dont il est question dans l'énoncé. On s'attachera un peu plus loin à rendre compte de ce type d'emploi.

Dans la TOPE, la subjectivité par défaut est celle de l'énonciateur. Le terme introduit par pour peut correspondre à une source de subjectivité qui lui est identifiable, ou, au contraire, qui s'inscrit en porte-à-faux par rapport à cet énonciateur, comme illustré en (13) et (14) :

(13) Pour Alain Badiou, la singularité n'est qu'une dimension du multiple, alors que pour moi, elle est le nom innommable.

(14) Pour Hegel, le mouvement de la pensée qu'il personnifie sous le nom de l’Idée, est le démiurge de la réalité, laquelle n'est que la forme phénoménale de l'Idée. Pour moi, au contraire, le mouvement de la pensée n’est qu'une réflexion du mouvement réel, transporté et transposé dans le cerveau de l'homme.

Dans ces énoncés, il est préférable de considérer que le terme Y correspond à un sujet-point de vue sur l'énoncé, et non à un énonciateur rapporté selon la définition qu'en donnent Hanote et Chuquet, 2004. Il ne se substitue pas à l'énonciateur, qui conserve ses prérogatives sur l'énoncé. En effet, si on s'intéresse à la prise en charge de la modalité, on s'aperçoit que, si la modalité radicale, qui constitue un jugement sur la mise en relation d'un sujet et d'un prédicat, relève de la responsabilité du référent de Y, la modalité épistémique peut, quant à elle, être prise en charge par l'énonciateur : 
(15) Pour lui, le plus grand danger qu'il faut conjurer coûte que coûte, c'est l'isolationnisme américain.

(16) Pour elle, je devais grandir le plus rapidement possible, non pas jouer ou chanter, mais parler et très vite lire, discuter avec elle de philosophie [...]

(17) Pour eux peut-être que c'est tout à fait naturel. Pourquoi faudrait-il qu'ils se conforment à nos valeurs?

(18) Et le voilà qui me pousse dans le couloir comme si j’étais dans un fauteuil roulant sauf que je ne suis pas dans un fauteuil roulant et que pour lui, ça doit faire une sacrée différence...

Les gloses ci-après font apparaître la différence entre (15) et (16) qui relèvent de la modalité radicale, d'une part, et (17) et (18), qui relèvent, eux, de la modalité épistémique :

(15’) Il pense que le plus grand danger qu'il faut conjurer coûte que coûte, c'est l'isolationnisme américain.

(16’) Elle estimait que je devais grandir le plus rapidement possible, non pas jouer ou chanter, mais parler et très vite lire, discuter avec elle de philosophie [...]

(17') Il se peut que, pour eux, ce soit tout à fait naturel [...]

(18') Et le voilà qui me pousse dans le couloir comme si j'étais dans un fauteuil roulant sauf que je ne suis pas dans un fauteuil roulant et il est probable que pour lui, ça fasse une sacrée différence...

Dans les énoncés manipulés (17’) et (18’), on a sorti la modalité épistémique de la relation prédicative et de la portée du syntagme prépositionnel sans modification majeure du sens de l'énoncé, ce qui confirme que cette modalité relève de l'énonciateur et non du référent de Y.

Ces éléments indiquent que, même si $\mathrm{Y}$ peut parfois être identifié à l'énonciateur, cette identification ne va pas de soi, puisque $Y$ est avant tout construit comme source de point de vue, et non comme énonciateur. En cela, pour se distingue d'autres marqueurs comme d'après, par exemple, qui introduit, lui, un énonciateur rapporté (au sens de Hanote et Chuquet 2004). Ainsi, par exemple, avec ce marqueur, c'est systématiquement le référent du terme Y qui prend en charge la modalité épistémique :

(19) D'après O'Meara, il est possible que l'Empereur ait eu une légère atteinte de scorbut.

(19’) O’Meara pense qu'il est possible que l'Empereur ait eu une légère atteinte de scorbut.

D’après construit vraisemblablement une altérité sur les énonciateurs, et non, comme le fait pour, sur les sujets-point de vue.

\section{Les ÉNONCÉS DU TYPE : POUR LE TOURISME, CE PAYS EST EN RETARD.}

L'altérité peut également porter sur le thème ou le topic (ce concept ne sera pas discuté dans le cadre de cet article, pour une discussion approfondie sur le sujet, on pourra se reporter à Berthoud 1996).

Dans ce cas également, la validation de la relation prédicative se trouve restreinte à un domaine $\mathrm{Y}$, que l'on pourra toujours opposer à un domaine ou une 
classe de domaines Y'. Ainsi, en (3), la propriété < être en retard> est attribuée au référent de ce pays uniquement dans le domaine représenté par le tourisme, et il est tout à fait possible de concevoir que le référent de ce pays ne possède pas cette propriété dans un autre domaine. C'est la même chose en (20), dans lequel la relation / les estimations de croissance pour cette année sont restées stables à $1 \%$ / n'est validée que dans le domaine délimité par la zone euro, et pas dans les domaines Y' les Etats-Unis, le Japon et la Chine.

(20) Grâce à un premier semestre meilleur que prévu, les perspectives ont donc été revues à la hausse pour la plupart des grandes économies, comme les EtatsUnis (à 3,3\%), le Japon (0,5\%) et la Chine (10,5\%). Pour la zone euro en revanche, les estimations de croissance pour cette année sont restées stables à $1 \%$.

Ces remarques s'appliquent aussi aux expressions du type de pour ce qui concerne :

(21) Pour ce qui est de la concision, je suis d'accord, mais ses phrases ne veulent rien dire.

(22) Voici vos billets, votre itinéraire, les adresses de nos agences à l'étranger. Pour ce qui est des repas, vous devrez les régler vous-mêmes sur place.

En (21), l'énonciateur limite son appréciation (je suis d'accord) au fait que le texte soit concis, ce qui est explicité par la présence de mais ses phrases ne veulent rien dire. De même, en (22), l'énonciateur mentionne les repas comme étant un élément dont chaque participant doit s'occuper par lui-même alors que les billets, etc. ont été préparés par l'énonciateur. On observe un phénomène similaire en (23) :

(23) Pour ce qui concerne l'achat de villas haut de gamme à Cannes et dans ses environs, il est indispensable de s'adresser directement aux meilleurs spécialistes.

Dans cet énoncé, trouvé sur le site d'une agence immobilière, il est question d'attirer l'attention des lecteurs sur l'achat de villas haut de gamme plutôt que sur l'achat d'autres biens immobiliers. Ici, il est sous-entendu que la relation / ilêtre indispensable de s'adresser directement aux meilleurs spécialistes / n'est pas validée dans un domaine autre que celui dénoté par l'achat de villas haut de gamme à Cannes et dans ses environs.

On a précisé plus haut que le terme Y pouvait renvoyer à un animé humain. Dans ce cas, comme dans les autres énoncés du même type, pour entre en concurrence avec le marqueur quant à :

(24) Ainsi commence leur amour. Coup de foudre pour lui. Pour elle, on ne sait pas.

(25) Le petit René ne donne pas de nouvelles et mes grands-parents s'assombrissent. Pour Pierre Marquis, mon cousin, on sait maintenant. Il est mort, tout à fait au début, en 1940, carbonisé dans un train qui a brûlé sur le pont de Kehl. 
(24') Ainsi commence leur amour. Coup de foudre pour lui. Quant à elle, on ne sait pas.

(25') Le petit René ne donne pas de nouvelles et mes grands-parents s'assombrissent. Quant à Pierre Marquis, mon cousin, on sait maintenant. Il est mort, tout à fait au début, en 1940, carbonisé dans un train qui a brûlé sur le pont de Kehl.

(24) implique deux animés humains, les référents de lui et d'elle. Dans le contexte à gauche du syntagme prépositionnel, l'énoncé correspond à une information concernant le référent de lui : il a eu le coup de foudre. Le syntagme prépositionnel introduit le passage à un nouveau thème, représenté par elle, pour qui on dit qu'on ne sait pas ce qu'il en est. (25) est similaire, le premier thème étant constitué par le petit René, et le second par le terme Y, Pierre Marquis, mon cousin.

Comme quant à, pour est compatible avec des effets de liste, d'après l'analyse de Choi-Jonin (2003) :

L'effet de liste est en effet nécessaire pour l'emploi de quant à en position frontale. Il permet d'isoler un élément discursif d'un ensemble défini qui en comporte d'autres. Quant à présuppose donc un ensemble défini, c'est-à-dire un ensemble déjà introduit dans une mémoire discursive. Le constituant qui suit quant à réfère donc à une partie de cet ensemble.

En effet, on observe que les entités qui sont introduites par pour ou par quant à s'inscrivent dans une sorte de paradigme avec les thématiques précédentes. La prudence s'impose cependant, cet effet paradigmatique pouvant tout à fait être lié à la nécessité de l'existence d'une cohérence discursive.

Ce qu'il est par contre intéressant de noter, c'est qu'il y a passage d'un premier thème / topic à un second thème / topic, construit en altérité avec le premier, ce qui semble être le cas avec les deux marqueurs. Encore une fois, le fonctionnement de pour est caractérisé par la construction d'un domaine $\mathrm{Y}^{\prime}$ en opposition à $\mathrm{Y}$, dans lequel la relation prédicative $\mathrm{X}$ n'est pas nécessairement validée.

\section{LES ÉNONCÉS DU TYPE : POUR L’INSTANT, IL ATTEND.}

Si on se tourne à présent vers le domaine de la temporalité, il convient de noter, d'emblée, que l'importance de la position détachée du syntagme prépositionnel apparaît de façon particulièrement marquée dans les contextes de cet ordre. Ainsi, la position détachée permet la présence en Y de termes qu'on ne retrouve pas en l'absence de détachement, ou entraîne des variations aspectuelles importantes d'un énoncé à l'autre. On comparera (26) et (27), d'une part, et (28) et (28') d'autre part :

(26) Nous viendrons peut-être le mois prochain mais c'est pas facile à cause du boulot. Pour le moment je suis avec ta mère et la tante Suzanne à faire des dessus de chaises pour la salle à manger pour pas abîmer nos brioches.

(27) Jean est hospitalisé pour deux semaines.

(28) Il s'est engagé chez Toyota pour deux ans. 
(28') Il s'est engagé chez Toyota, pour l'instant. / Pour l'instant, il s'est engagé chez Toyota.

En (27) et (28), deux semaines et deux ans correspondent respectivement à la durée (certes encore virtuelle en $\mathrm{T}_{2}$, au moment de l'événement) de l'hospitalisation ou de l'engagement dont il est question dans l'énoncé. En (28'), par contre, il n'est pas question de mesurer la durée de l'événement, mais de l'opposer à une situation autre :

(28’') Il s'est engagé chez Toyota en attendant / faute de mieux / mais il peut revenir sur sa décision.

Dans les énoncés (26) et (28), le nom d'unité est introduit par l'article défini. Or, celui-ci entretient des liens privilégiés avec pour dans ses emplois temporels en position détachée, y compris avec des noms d'unités. Pour l'instant et pour le moment sont, en effet, très fréquents, alors que pendant, qui est susceptible d'entrer en concurrence avec ce marqueur dans certains de ses emplois temporels, ne fonctionne pas en combinaison avec ces termes.

Un aspect particulier du fonctionnement de l'article défini est qu'il introduit une occurrence dont les délimitations qualitative et quantitative sont stabilisées. Cela implique que cette occurrence possède des propriétés différentielles qui la rendent distinguable de toute autre occurrence de la même classe. Ainsi, dans pour l'instant ou pour le moment, il est question d'un instant / moment particulier (ici $\mathrm{T}_{0}$ ), qui se construit en altérité par rapport à tout autre membre de la classe des /instant/ ou des /moment/. Autrement dit, ce n'est pas l'instant ou le moment (ou la semaine, ou le mois) en tant qu'unité de temps qui compte, mais en tant qu'il possède des propriétés remarquables permettant de le pointer et de le distinguer d'un instant autre.

Ces affinités de la position détachée avec le fléchage nous semblent aller également dans le sens d'une hypothèse d'analyse en termes de hiatus pour le marqueur pour, la validation de la relation prédicative $\mathrm{X}$ étant restreinte à Y.

L'examen sommaire de ces quatre emplois de pour va dans le sens de nos hypothèses de départ, qui étaient, rappelons-le, (i) que le marqueur introduit un terme $\mathrm{Y}$ qui s'interprète comme le domaine dans lequel la relation prédicative $\mathrm{X}$ est validée, (ii) et que le terme $\mathrm{Y}$ est systématiquement construit en opposition par rapport à un autre domaine (Y'), dans lequel c'est $\mathrm{X}^{\prime}$ (c'est-à-dire non-X) qui est validé. Dans les énoncés que nous avons analysés, il est remarquable que l'opération d'altérité dont pour est la trace (cf Homma 2009 et Hamelin 2010b) se manifeste par la construction de domaines qui se définissent négativement par rapport à Y (non Y), et que ce type de fonctionnement s'observe essentiellement dans les domaines de l'aspectualité (temps et point de vue), de la topicalisation, et du commentaire linguistique. Voilà qui invite à s'interroger sur la relation que peuvent entretenir, dans le cadre de la TOPE, l'opération de repérage avec les phénomènes de détachement. Par ailleurs, on a souligné en particulier les affinités de l'emploi de pour en position initiale détachée avec le fléchage au moyen de l'article défini. Il apparaît que ce marqueur permet la construction d'un terme Y possédant des propriétés différentielles, compatibles avec la construction d'une occurrence ou d'une classe d'occurrences en altérité avec ce terme Y. La 
fréquence de ce type de détermination dans les énoncés envisagés renforce encore l'analyse du marqueur pour en termes de construction d'un hiatus sur Y proposée dans ces pages.

\section{BIBLIOGRAPHIE}

Berthoud A.C., 1996, Paroles à propos. Approche énonciative et interactive du topic, Paris, Ophrys.

Choi-Jonin I., 2003, Ordre syntaxique et ordre référentiel : emplois de la locution prépositive quant à, in B. Combette, C. Schnedecker \& A. Theissen (éds), Ordre et distinction dans la langue et le discours, Actes du Colloque international de Metz (18, 19, 20 mars 1999), Paris, Honoré Champion éditeur, p. 133-147.

Culioli A., 1990, Pour une linguistique de l'énonciation, Opérations et représentations, Tome 1 , Gap, Ophrys.

Culioli A., 1999a, Pour une linguistique de l'énonciation, Formalisation et opérations de repérage, Tome 2, Gap, Ophrys.

Culioli A., 1999b, Pour une linguistique de l'énonciation, Domaine notionnel, Tome 3, Gap, Ophrys.

Dufaye L., 2009, I wrote this article in the space of a week in et since/for : analyse topologique des emplois temporels, in C. Delmas (éd.), Temps et/ou espace, Grammaire et/ou Grammaticalisation, Actes du colloque de Monbazillac des 29-30 septembre 2006, Paris, Presses de la Sorbonne Nouvelle, p. XXXXX.

Dufaye L., 2007, Localisation spatiale et temporelle avec in, on et at, Complétude, cognition, construction linguistique, in C. Delmas (éd.), Temps et/ou espace, Grammaire et/ou Grammaticalisation, Actes du colloque de Monbazillac des 29-30 septembre 2006, Paris, Presses de la Sorbonne Nouvelle,, p. 99-109.

Gilbert E., 2000, For et la construction des espaces référentiels, in C. Guimier (ed), Syntaxe et sémantique-connecteurs et marqueurs de connexion, Presses Universitaires de Caen, $\mathbf{p}$. XXXXX.

Gilbert E., 2004, Ebauche d'une formalisation des prépositions in, on et at, Cycnos 21-1, p. 93-111.

Gilbert E., 2006, Remarque autour de quelques prépositions, in D. Ducard \& C. Normand (dir.), Antoine Culioli, un homme dans le langage, actes du colloque de Cerisy-la-salle, Juin 2005, p. XXXXX.

Gilbert E., 2007, A propos de behind et after, Syntaxe et Sémantique8, Presses Universitaires de Caen, p. XXXXX.

Franckel J.-J. \& Paillard D., 2007, Grammaire des prépositions, tome 1, Paris, Ophrys, Collection "L’homme dans la langue".

Hamelin L., 2010a, To me, chess is not a sport: to et le point de vue, Corela 8-2,

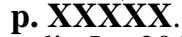

Hamelin L., 2010b, A propos de pour, quelques remarques sur l'expression de la cause et du but, Actes du CMLF 2010. A COMPLETER

Hanote S. \& Chuquet H., 2004, 'Who's speaking, please ?' - Le discours rapporté, Gap, Ophrys, Collection GRAMVOC.

Homma Y., 2009, L'identité des prépositions dans leur variation : approche énonciative de en, dans, pour et par, Thèse de Doctorat.

Neveu F., 2004, Dictionnaire des Sciences du langage, Paris, Armand Colin. 